\title{
Kufor-Rakeb syndrome
}

INSERM

\section{Source}

INSERM. (1999). Orphanet: an online rare disease and orphan drug data base. KuforRakeb syndrome. ORPHA:306674

Kufor-Rakeb syndrome (KRS) is a rare genetic neurodegenerative disorder characterized by juvenile Parkinsonism, pyramidal degeneration (dystonia), supranuclear palsy, and cognitive impairment. 